\title{
Two-Dimensional DOA Estimation of MIMO Radar Coherent Source Based on Toeplitz Matrix Set Reconstruction
}

\author{
Fei Zhang $\mathbb{D}^{\mathrm{D}}$, Aisuo Jin, and Yin Hu \\ School of Electronic Information, Jiangsu University of Science and Technology, Zhenjiang, Jiangsu 212000, China \\ Correspondence should be addressed to Fei Zhang; zjzf@just.edu.cn
}

Received 14 October 2020; Revised 23 November 2020; Accepted 30 December 2020; Published 4 February 2021

Academic Editor: Xingwang Li

Copyright (c) 2021 Fei Zhang et al. This is an open access article distributed under the Creative Commons Attribution License, which permits unrestricted use, distribution, and reproduction in any medium, provided the original work is properly cited.

\begin{abstract}
In order to realize the high-precision direction of arrival (DOA) estimation of the coherent source of two-dimensional multipleinput and multiple-output (MIMO) radar, a solution is given by combining Toeplitz matrix set reconstruction. MIMO radar obtains a larger aperture with fewer arrays. Traditional two-dimensional reconstruction Toeplitz-like algorithms use part of the information in the construction of two correlation matrices or covariance matrices to construct the Toeplitz matrix when performing two-dimensional coherent source DOA estimation, which makes the information utilization incomplete and requires additional denoising processing. To solve the above problems, this paper proposes an improved Toeplitz matrix set reconstruction algorithm based on the two-dimensional reconstruction Toeplitz class algorithm. The complete array element receiving signal vector is used to construct two Toeplitz matrix sets containing complete information, and then their conjugate transposes. Multiply and sum to correct the matrix to obtain a full-rank matrix, so as to achieve the purpose of decoherence and combine the traditional ESPRIT algorithm to perform two one-dimensional reconstruction processing through rotation invariance and then perform angle matching to achieve two-dimensional coherent signal angle estimation, while avoiding additional denoising processing. Finally, the simulation results of the cross array and the $L$-shaped array verify the effectiveness of the algorithm in this paper and further extend it to the two-dimensional MIMO radar array model and compare it with the traditional ESPRIT-like algorithm and the REC-FBSS-ESPRIT algorithm. In comparison, the algorithm in this paper has better performance under the conditions of successful resolution, DOA estimation accuracy, and low signal-to-noise ratio.
\end{abstract}

\section{Introduction}

The multiple-input and multiple-output (MIMO) radar system is a new radar system proposed in recent years. MIMO technology has brought a new breakthrough to the application performance of the radar system. Compared with the traditional radar, MIMO radar has potential advantages in parameter target estimation, target parameter detection, estimation performance analysis, space-time adaptive processing suppression, radar interference waveform design, and so forth. The direction of arrival (DOA) [1] estimation problem is one of the important research directions in the signal processing of sensor arrays $[2,3]$, and it is widely used in radar [4-6]. High-precision DOA estimation based on subspace has become the focus of research [7-9]; for example, multiple signal classification (MUSIC) and estimation of signal parameters via rotational invariance techniques (ESPRIT) [10] can provide higher resolution to estimate the direction of arrival of uncorrelated and partially related signals [11-13]. But in practice, there are a large number of coherent sources due to multipath propagation and cofrequency interference [14]. When the signals are coherent, the rank of the covariance matrix accepted by the array will be deficient, so that the signal will be diffused to the noise, and the performance of the DOA estimation will be degraded $[15,16]$. The methods of decoherence include spatial smoothing algorithms, such as forward smoothing, backward smoothing, and forward and backward smoothing, but they are usually only applicable to isometric uniform linear arrays, and the corrected dimension is lower than the original matrix dimension; that is, decoherence is in exchange for lower degrees of freedom $[6,14]$. 
Another problem to solve rank deficit is to construct an algorithm based on vector and matrix reconstruction [14]. This algorithm rearranges the elements by covariance processing of the vector of the received signal and then constructs a Toeplitz matrix so that its rank is only the direction of arrival is related without being affected by signal correlation, so as to achieve the purpose of decoherence. Reference [17] proposed the ESPRIT-like algorithm by constructing a special antenna array model to reconstruct a Toeplitz matrix with the covariance matrix of the received signal matrix and using rotation invariance to achieve decoherence, but this method only works for the center array of the covariance matrix. When the center row of the covariance matrix is selected for reconstruction, the noise term is only a scalar quantity; otherwise, the denoising process still needs to be used to estimate the DOA using the ESPRIT algorithm. Because only one line of covariance is used, its information utilization is incomplete and affects DOA estimation performance. Reference [6] extended [17] to the two-dimensional DOA estimation of coherent sources. Reference [18] proposed the REC-FBSS-ESPRIT algorithm for reconstructing a covariant matrix, using the forward and backward spatial smoothing method as a preprocessing method to solve the problem of rank deficiency, and constructed an L-shaped matrix for two-dimensional DOA estimation, but the signal is still affected by noise and requires denoising. Reference [14] proposed an improved algorithm based on Toeplitz matrix reconstruction, by constructing a Toeplitz matrix set on the signal matrix, processing the Toeplitz matrix and the signal matrix, and then multiplying and summing by the conjugate transposed matrix. The matrix is corrected to obtain a full-rank matrix to achieve decoherence, and no additional denoising is required, but it is only suitable for one-dimensional DOA estimation.

Based on the literature [14], this paper proposes an improved Toeplitz matrix set reconstruction algorithm based on the two-dimensional reconstruction of the Toeplitz-like algorithms and uses cross arrays and $L$-shaped arrays for the two-dimensional DOA estimation [19], further extending to MIMO radar two-dimensional DOA estimation, using the characteristics of MIMO radar, with fewer array antennas to obtain higher DOA measurement accuracy. The algorithm uses two even linear arrays of mutually orthogonal array elements, through two permutation matrices and the relationship between the signal subspace and the direction vector, using rotation invariance, through two one-dimensional processing and then angle matching. Thus, DOA estimation of two-dimensional coherent signals is realized. Finally, simulation experiments verify the universality of the proposed algorithm for two-dimensional DOA estimation. Compared with the traditional ESPRIT-like algorithm and the REC-FBSS-ESPRIT algorithm in [18], the successful resolution probability and DOA estimation accuracy of the algorithm in this paper have better performance in the case of low signal-to-noise ratio, does not require spectrum peak search, and the amount of calculation is small.

\section{Materials and Methods}

2.1. Cross Array. A cross-shaped array as shown in Figure 1 supposes the intersection of the cross-shaped array is the origin of the coordinates, the array elements are evenly distributed on the $x$-axis and $y$-axis, and the number of array elements on the $x$-axis and $y$-axis is $N=2 M+1$. The total number of array elements is $4 M+1$. The array element spacing is $d=(\lambda / 2)$, where $\lambda$ is the wavelength. Assuming that $P$ signals are incident on the antenna, the two-dimensional arrival angles of the $i$ th signal are $\theta i, \phi i(i=1,2$, $\ldots, P)$, and $\theta i$ and $\phi i$ are the azimuth angle and the pitch angle, respectively.

Then, the received signals of $x$-axis and $y$-axis are

$$
\left\{\begin{array}{l}
X=A_{x} s+N_{x}, \\
Y=A_{x} s+N_{x},
\end{array}\right.
$$

where $A_{x}=\left[a_{x}\left(\theta_{1}\right), a_{x}\left(\theta_{2}\right), \ldots, a_{x}\left(\theta_{p}\right)\right] \quad$ and $A_{y}=\left[a_{y}\left(\theta_{1}\right), a_{y}\left(\theta_{2}\right), \ldots, a_{y}\left(\theta_{p}\right)\right]$ are the $x$-axis and $y$-axis direction matrices, respectively; the dimension is $(2 \mathrm{M}+1) \times$ $\mathrm{P}, \quad$ and $a_{x}\left(\theta_{p}\right)=\left[e^{-j 2 \pi M d \sin \theta_{p} \sin }\right.$

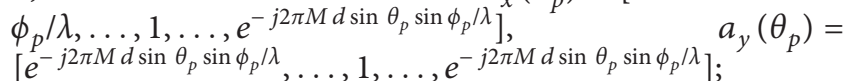
$s=\left[s_{1}, s_{2}, \ldots, s_{p}\right]$ is $P \times 1$ dimension incident signal vector; $P$ signals can be independent, correlated, or coherent; $N_{x}$ and $N_{y}$ are the additive Gaussian noise of the $x$-axis and $y$-axis receiving models, respectively; the dimensions are $2 M+1$; and they are not related to the source.

First, construct the Toeplitz matrices and of the received signal. As can be seen from [14], Toeplitz matrices $B_{x}(t)$ and $B_{y}(t)$ can be expressed as follows:

$$
\left\{\begin{aligned}
B_{x}(t) & =\left[\begin{array}{cccc}
X_{0}(t) & X_{1}(t) & \cdots & X_{M}(t) \\
X_{-1}(t) & X_{0}(t) & \cdots & X_{M-1}(t) \\
\vdots & \vdots & \ddots & \vdots \\
X_{-M}(t) & X_{-M+1}(t) & \cdots & X_{0}(t)
\end{array}\right]=A_{r x} S A_{r x}^{H}+B_{N x}(t), \\
B_{y}(t) & =\left[\begin{array}{cccc}
Y_{0}(t) & Y_{1}(t) & \cdots & Y_{M}(t) \\
Y_{-1}(t) & Y_{0}(t) & \cdots & Y_{M-1}(t) \\
\vdots & \vdots & \ddots & \vdots \\
Y_{-M}(t) & Y_{-M+1}(t) & \cdots & Y_{0}(t)
\end{array}\right]=A_{r y} S A_{r y}^{H}+B_{N y}(t),
\end{aligned}\right.
$$




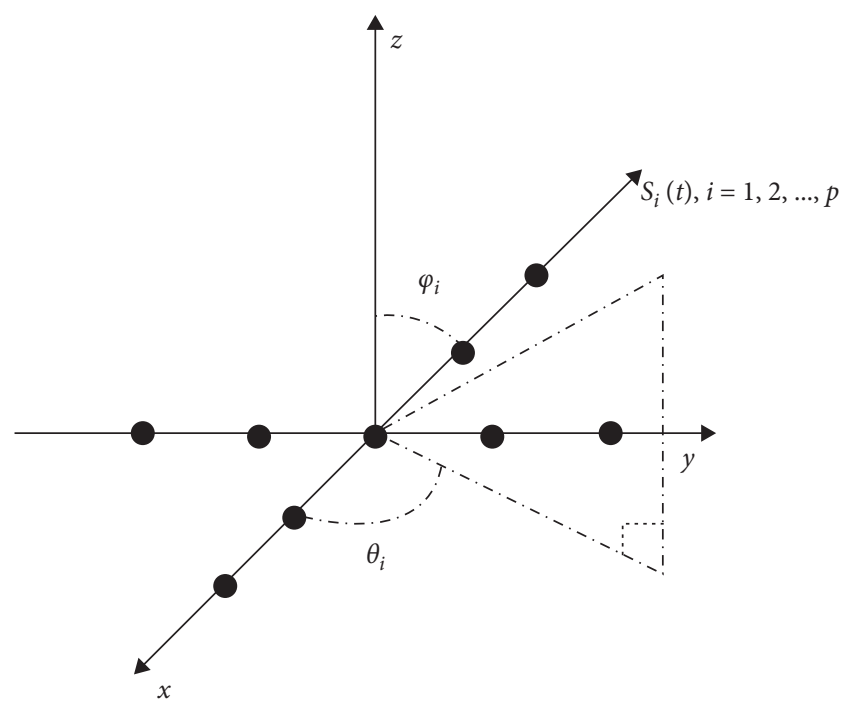

Figure 1: Cross array.

where $\quad A_{r x}=\left[a_{r x}(\theta 1), a_{r x}(\theta 2), \ldots, a_{r x}\left(\theta_{p}\right)\right]$, $a_{r x}\left(\theta_{p}\right)=\left[1, e^{-j 2 \pi d \sin \theta_{p} \sin \phi_{p} / \lambda}, \ldots, e^{-j 2 \pi d \sin \theta_{p} \sin \phi_{p} / \lambda}\right]$, and $a_{r y}\left(\theta_{p}\right)=\left[1, e^{-j 2 \pi d \cos \theta_{p} \sin \phi_{p} / \lambda}, \ldots, e^{-j 2 \pi d \cos \theta_{p} \sin \phi_{p} / \lambda}\right]$ in the same way; $S=\operatorname{diag}\{s 1, s 2, \ldots, s p\}$ which means that $S$ is a full-rank diagonal matrix. In other words, the rank of $S$ is independent of the coherence between signals, and the decorrelation can be achieved. $B_{N x}(t)$ and $B_{N y}(t)$ are Toeplitz matrices composed of noise vectors.

2.2. L-Shaped Array. L-shaped array is shown in Figure 2: let the coordinate origin of the $L$-shaped array be the reference array element. The array elements are evenly distributed on the $x$-axis and the $y$-axis. The numbers of array elements on the $x$-axis and the $y$-axis are both $N=2 M+1$ and the total number of array elements is $4 M+1$. The array element spacing is $d=(\lambda / 2)$, where $\lambda$ is the wavelength. Assuming that $P$ signals are incident on the antenna, the two-

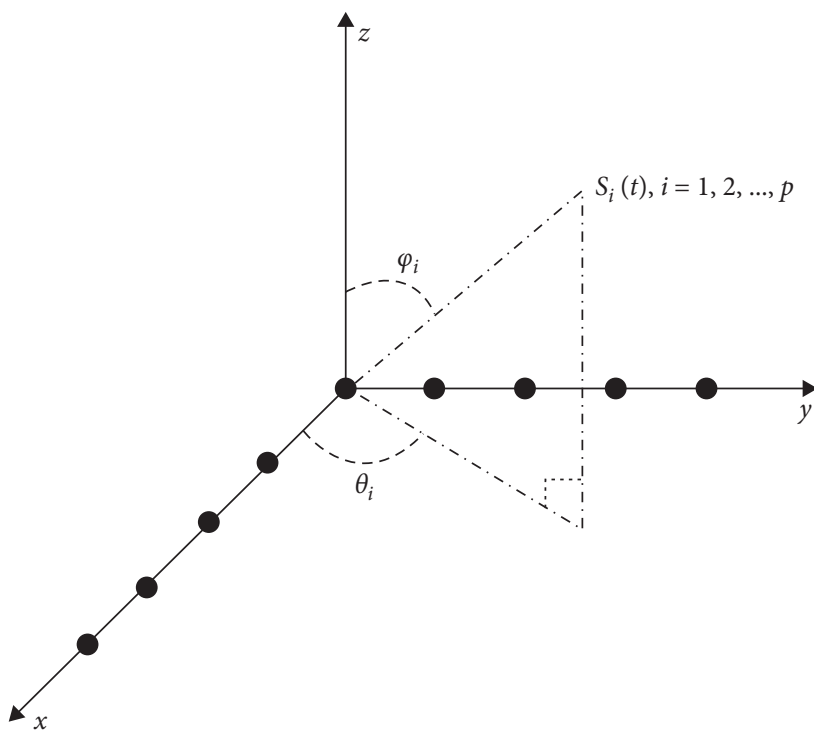

FIgURE 2: $L$-shaped array.

dimensional arrival angles of the $i$ th signal are $\theta i, \phi i(i=1,2$, $\ldots, P)$, and $\theta i$ and $\phi i$ are the azimuth angle and the pitch angle, respectively.

Similarly, the received signals of the $x$-axis and $y$-axis are

$$
\left\{\begin{array}{l}
X=A_{x} s+N_{x} \\
Y=A_{x} s+N_{x}
\end{array}\right.
$$

In the same way, where $A x$ and $A y$ are the direction matrix of the $x$-axis and $y$-axis, respectively, the dimension is $(2 M+1) \times P, \quad$ where $a_{x}\left(\theta_{p}\right)=\left[1, e^{\left(j 2 \pi d \sin \theta_{p} \sin \phi_{p} / \lambda\right)}, \ldots, e^{\left(j 2 \pi M d \sin \theta_{p} \sin \phi_{p} / \lambda\right)}\right]$; $a_{y}\left(\theta_{p}\right)=\left[1, e^{\left(j 2 \pi d \cos \theta_{p} \sin \phi_{p} / \lambda\right)}, \ldots, e^{\left(j 2 \pi M d \cos \theta_{p} \sin \phi_{p} / \lambda\right)}\right]$; and $S$ and $N$ are the same as the cross array.

The Toeplitz matrices $B_{x}(t)$ and $B_{y}(t)$ are constructed as follows:

$$
\left\{\begin{array}{c}
B_{x}(t)=\left[\begin{array}{cccc}
X_{M}(t) & X_{M+1}(t) & \cdots & X_{2 M}(t) \\
X_{M-1}(t) & X_{M}(t) & \cdots & X_{2 M-1}(t) \\
\vdots & \vdots & \ddots & \vdots \\
X_{0}(t) & X_{1}(t) & \cdots & X_{M}(t)
\end{array}\right]=C_{1} A_{r x} S A_{r x}^{H}+B_{N x}(t), \\
B_{y}(t)=\left[\begin{array}{cccc}
Y_{M}(t) & Y_{M+1}(t) & \cdots & Y_{2 M}(t) \\
Y_{M-1}(t) & Y_{M}(t) & \cdots & Y_{2 M-1}(t) \\
\vdots & \vdots & \ddots & \vdots \\
Y_{0}(t) & Y_{1}(t) & \cdots & Y_{M}(t)
\end{array}\right]=C_{2} A_{r y} S A_{r y}^{H}+B_{N y}(t) .
\end{array}\right.
$$

Among them, $C_{1}$ and $C_{2}$ are constants; since $S$ is a diagonal matrix, the correlation matrix is not affected by the constants. If $S=C S$, then $B_{x}(t)$ and $B_{y}(t)$ are the same.
2.3. MIMO Array. The single-static MIMO array is shown in Figure 3; set the number of transmitting array elements as $1 \times M$ and the number of receiving array elements as $N \times 1$; 


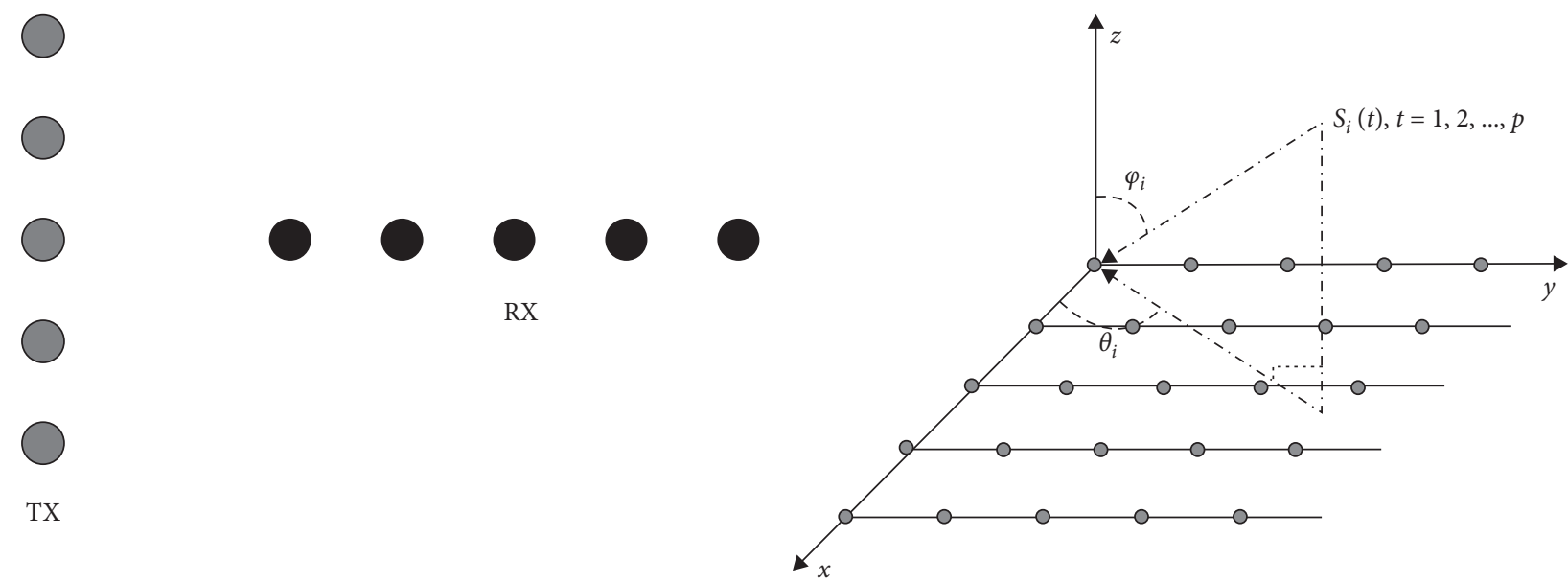

(a)

(b)

FIgURE 3: MIMO array. (a) Transmitting and receiving array model. (b) Virtual array model.

that is, the total number of array elements is $M+N$. Due to the nature of the MIMO array model, it can be virtualized. The number of arrays is $M \times N$, and the array elements are uniformly distributed on the $x$-axis and $y$-axis, respectively, and the total number of array elements is $M \times N$. The element spacing is $d=(\lambda / 2)$, where $\lambda$ is the wavelength. Assuming that $P$ signals are incident on the antenna, the twodimensional arrival angles of the $i$ th signal are $\theta i, \phi i(i=1,2$, $\ldots, P)$, and $\theta i$ and $\phi i$ are the azimuth and elevation angles, respectively.

Then, the output is

$$
\begin{aligned}
x(l)= & {\left[a_{t}\left(\theta_{1}, \phi_{1}\right) \otimes a_{r}\left(\theta_{1}, \phi_{1}\right), a_{t}\left(\theta_{2}, \phi_{2}\right) \otimes a_{r}\left(\theta_{2}, \phi_{2}\right), \ldots,\right.} \\
& \left.a_{t}\left(\theta_{k}, \phi_{k}\right) \otimes a_{r}\left(\theta_{k}, \phi_{k}\right)\right] S(l)+n(l),
\end{aligned}
$$

where $\theta_{k}, \phi_{k}$ are the elevation and azimuth angles corresponding to the $k$ th target; $n(l)$ is the $M N \times 1$ Gaussian white noise vector, the mean is 0 , and the covariance is $\sigma 2 I_{M N}$; and at $\left(\theta_{k}, \phi_{k}\right)=a_{t y}\left(\theta_{k}, \phi_{k}\right) \otimes a_{t x}\left(\theta_{k}, \phi_{k}\right) \quad$ and $a_{r}\left(\theta_{k}, \phi_{k}\right)=a_{r y}\left(\theta_{k}, \phi_{k}\right) \otimes a_{r x}\left(\theta_{k}, \phi_{k}\right)$, where $a_{t y}\left(\theta_{k}, \phi_{k}\right)$ and $a_{t x}\left(\theta_{k}, \phi_{k}\right)$ are the antenna steering vectors along the $y$-axis and $x$-axis of the transmitting array (corresponding to the $k$ th target $)$ and $a_{r y}\left(\theta_{k}, \phi_{k}\right)$ and $a_{r x}\left(\theta_{k}, \phi_{k}\right)$ are the same. So, there are the following relations

$$
\left\{\begin{array}{l}
a_{t y}\left(\theta_{k}, \phi_{k}\right)=[1,1, \ldots, 1], \\
a_{t x}\left(\theta_{k}, \phi_{k}\right)=\left[1, e^{-j \pi \sin \theta_{k} \cos \phi_{k}}, \ldots, e^{-j M \pi \sin \theta_{k} \cos \phi_{k}}\right], \\
a_{r y}\left(\theta_{k}, \phi_{k}\right)=\left[1, e^{-j \pi \sin \theta_{k} \sin \phi_{k}}, \ldots, e^{-j N \pi \sin \theta_{k} \sin \phi_{k}}\right], \\
a_{r x}\left(\theta_{k}, \phi_{k}\right)=[1,1, \ldots, 1] .
\end{array}\right.
$$

Then, set $x(l)$ to $X=A_{x} s+N_{x}$. The same is $Y=A_{y} s+N_{y}$.

Correlation calculation of $B_{x}(t)$ and $B_{y}(t)$ with the corresponding received signals $X_{i}(t)$ and $Y_{i}(t)$ of the $i$ th array element:

$$
\left\{\begin{array}{l}
R_{X i}=E\left[B_{x}(t) X_{i}^{*}(t)\right]=A_{r x} E\left[S x_{i}^{*}(t)\right] A_{r x}^{H}+\sigma_{n}^{2} I_{x,(M+1), i} \\
=R_{x i}+\sigma_{n}^{2} I_{x,(M+1), i} \\
R_{Y i}=E\left[B_{y}(t) Y_{i}^{*}(t)\right]=A_{r y} E\left[S y_{i}^{*}(t)\right] A_{r y}^{H}+\sigma_{n}^{2} I_{y,(M+1), i} \\
=R_{y i}+\sigma_{n}^{2} I_{y,(M+1), i} .
\end{array}\right.
$$

Let $E\left[S x_{i}^{*}(t)\right]=S_{x i}$ and $E\left[S y_{i}^{*}(t)\right]=S_{y i}$, where $R_{x i}$ and $R y i$ are correlation operations without noise; $I_{M+1, i}$ is the $(M+1) \times(M+1)$-dimensional matrix whose $i$ th diagonal is 1. When $i=0, I_{M+1,0}=I_{M+1}$ is the $M+1$ dimension identity matrix; $E[\bullet]$ represents mathematical expectation.

It can be obtained from equation (7) that, for the ESPRITlike algorithm in [17], the method is to use an optional set of Toeplitz matrices $R_{X i}$ and $R_{Y i}$ to achieve the purpose of decoherence of coherent source signals. If $i=0$ is selected as the central array element to construct $R_{X i}$ and $R_{Y i}$, then $I_{M+1, i}$ is an identity matrix, and after that the relationship between the signal subspace and the direction vector can be used to directly perform feature decomposition on $R_{X i}$ and $R_{Y i}$ to find the final DOA information, but when $i \neq 0$ is the central array element, $I_{M+1, i}$ is not an identity matrix, so denoising is still required. And ESPRIT-like only constructs one $R_{X i}$ and $R_{Y i}$, so the complete information of the array is not fully utilized. To solve the above problems, all $R_{X i}, R_{Y i}$ and their corresponding $R_{X i}^{H}, R_{X i}^{H}$ are multiplied and summed:

Cross array:

$$
\left\{\begin{array}{c}
R_{X \Sigma}=A_{r x} \sum_{k=0}^{M} D_{x k}\left(\sum_{i=-M}^{M} C_{x i}+2 \sigma_{n}^{2} R_{s}\right) D_{x k}^{H} A_{r x}^{H} \\
+(M+1) \sigma_{n}^{4} I_{x, M+1,0}, \\
R_{Y \Sigma}=A_{r y} \sum_{k=0}^{M} D y k\left(\sum_{i=-M}^{M} C_{y i}+2 \sigma_{n}^{2} R_{s}\right) D_{y k}^{H} A_{r y}^{H} \\
\quad+(M+1) \sigma_{n}^{4} I_{y, M+1,0} .
\end{array}\right.
$$


L-shaped array:

$$
\left\{\begin{array}{l}
R_{X \Sigma}=A_{r x} \sum_{k=0}^{M} D_{x k}\left(\sum_{i=0}^{2 M} C_{x i}+2 \sigma_{n}^{2} R_{s}\right) D_{x k}^{H} A_{r x}^{H} \\
+(M+1) \sigma_{n}^{4} I_{x, M+1,0}, \\
R_{Y \Sigma}=A_{r y} \sum_{k=0}^{M} D_{y k}\left(\sum_{i=0}^{2 M} C_{y i}+2 \sigma_{n}^{2} R_{s}\right) D_{y k}^{H} A_{r y}^{H} \\
+(M+1) \sigma_{n}^{4} I_{y, M+1,0} .
\end{array}\right.
$$

The same is for the MIMO array.

$R_{X \Sigma}$ and $R_{Y \Sigma}$ obviously have a joint diagonalization structure and span the same range of space of $A_{r x}$ and $A_{r y}$, where $R_{s}=E\left[S S^{H}\right]$ is the signal covariance matrix. For $D_{x k}$, $D_{y k}, C_{x i}$, and $C_{y i}$, see literature [14].

From equations (8) and (9), it can be seen that the reconstructed equivalent covariance matrix is a positive definite matrix with $\operatorname{rank} P$. The rank is not affected by signal correlation, but only related to the number of signals [14]. To further improve accuracy, $R_{X \Sigma}$ and $R_{Y \Sigma}$ are revised

$$
\left\{\begin{array}{l}
R_{x}=\frac{1}{2}\left(R X \Sigma+J R_{X}^{*} \Sigma^{J}\right), \\
R_{y}=\frac{1}{2}\left(R_{Y \Sigma}+J R_{Y}^{*} \sum^{J}\right),
\end{array}\right.
$$

where $J$ is a permutation matrix with an antidiagonal of 1 . Since $A_{r x}$ and $A_{r y}$ are Vandermonde matrices and $\theta i \neq \theta_{j}$, $A_{r x}$ and $A_{r y}$ are full ranks, and because $\sum_{k=0}^{M} D_{x}^{k}\left(\sum_{i=-1}^{M} C_{x}+\right.$ $\left.2 \sigma_{n}^{2} R_{s}\right)\left(D_{x}^{k}\right)^{H}$ and $\sum_{k=0}^{M} D_{x}^{k}\left(\sum_{i=0}^{2 M} C_{x}+2 \sigma_{n}^{2} R_{s}\right)\left(D_{x}^{k}\right)^{H}$ are diagonal matrices, their feature decomposition can obtain $P$ large eigenvalues and $M+1-P$ small eigenvalues, where the eigenvectors corresponding to large eigenvalues constitute the signal subspace $\Phi_{s}=\operatorname{span}\left\{v_{1}, v_{2}, \ldots, v_{p}\right\}$ and the eigenvectors corresponding to the small eigenvalues constitute the noise subspace $\Phi_{s}=\operatorname{span}\left\{v_{1+p}, v_{2+p}, \ldots, v_{M}\right\}$.

\section{Two-Dimensional DOA Estimation of Coherent Sources}

Let $A_{r x}=\left[\begin{array}{c}a_{1} \\ A_{B x}\end{array}\right]=\left[\begin{array}{c}A_{F x} \\ a_{M+1}\end{array}\right]$, where $a_{1}$ and $a_{M+1}$ are the first and last terms of $A_{r x}$. Similarly, the signal subspace is decomposed $\left\{v_{1}, v_{2}, \ldots, v_{p}\right\}=U_{S x}=\left[\begin{array}{c}u_{1} \\ U_{B x}\end{array}\right]=\left[\begin{array}{c}U_{F x} \\ u_{M+1}\end{array}\right]$, where $u_{1}$ and $u_{M+1}$ are the first and last columns of $U_{S x}$, respectively. It is easy to get $A_{B x}=A_{F x} \Phi_{x}$, where

$$
\begin{aligned}
\Phi_{x}= & \operatorname{diag}\left\{e^{-j 2 \pi d \sin \theta_{1} \sin \phi_{1} / \lambda} e^{-j 2 \pi d \sin \theta_{2} \sin \phi_{1} / \lambda}\right. \\
& \left.\cdots e^{-j 2 \pi d \sin \theta_{p} \sin \phi_{1} / \lambda}\right\} .
\end{aligned}
$$

It is a rotation matrix. Since $A_{r x}$ and $U_{S x}$ are the same signal subspace, $U_{F x}=A_{F x} T$ and $U_{B x}=A_{B x} T$, in which $T$ is a full-rank matrix; then,

$$
U_{F x} T^{-1} \Phi_{x} T=A_{F x} T T^{-1} \Phi_{x} T=A_{F x} \Phi_{x} T=A_{B x} T=U_{B x}
$$

Let $\Psi_{x}=T^{-1} \Phi_{x} T$; then, $U_{F x} \Psi_{x}=U_{B x}$. We can derive $e^{-\left(j 2 \pi d \sin \theta_{k} \sin \phi_{k} / \lambda\right)}, k=1,2, \ldots, p$, by feature decomposition of $\Psi_{x}$. Both $\sin \theta_{k} \sin \phi_{k}$ can be estimated, and $\cos \theta_{k} \sin \phi_{k}$ can be estimated in the same way. The following two-dimensional DOA angle can be estimated:

$$
\left\{\begin{array}{l}
\theta_{k}=\tan ^{-1}\left(\frac{\sin \theta_{k} \sin \phi_{k}}{\cos \theta_{k} \sin \phi_{k}}\right), \\
\phi_{k}=\sin ^{-1}\left(\sqrt{\left(\sin \theta_{k} \sin \phi_{k}\right) 2+\left(\cos \theta_{k} \sin \phi_{k}\right) 2}\right) .
\end{array}\right.
$$

In summary, the steps of the two-dimensional DOA estimation method based on the Toeplitz matrix set proposed in this paper are shown in Table 1.

\section{Computer Simulation}

In order to verify the universality of the proposed algorithm for two-dimensional DOA estimation, the proposed algorithm is compared with the cross-shaped array ESPRIT-like algorithm in [6] and the L-shaped array REC-FBSS-ESPRIT algorithm in [18]. Perform 100 Monte Carlo simulations on each algorithm and define the root mean square error as

$$
\operatorname{RSME}=\frac{1}{K} \sum_{K=1}^{K} \sqrt{\frac{1}{100} \sum_{n=1}^{100}\left[\left(\widehat{\theta}_{k, n}-\theta_{k}\right) 2+\left(\widehat{\phi}_{k, n}-\phi_{k}\right) 2\right] .}
$$

The DOA of the three sources used in the simulation are $\left(35^{\circ}, 40^{\circ}\right),\left(75^{\circ}, 70^{\circ}\right),\left(89^{\circ}, 80^{\circ}\right)$, and sources 1,3 are related signals. The numbers of array elements on the $x$-and $y$-axis are both 11, and the array element spacing is half a wavelength.

Figure 4 shows the DOA estimation performance of each algorithm, where Figures 4(a)-4(e) are the cross-shaped ESPRIT-like algorithm, $L$-shaped array REC-FBSS-ESPRIT algorithm, MIMO array, and the DOA estimation results of the cross array, L-type array, and MIMO array using the algorithm of this paper when the signal-to-noise ratio is $\mathrm{SNR}=10$ and the number of snapshots is 1024 . From the figure, it can be seen intuitively that the algorithm of this paper is closer to the actual angle and has better two-dimensional DOA estimated performance.

Figure 5 shows the performance comparison of azimuth estimation using the cross array, $L$-shaped array, MMO array, ESPRIT-like algorithm, and REC-FBSS-ESPRIT algorithm using this algorithm and examines the RSME variation of the algorithm under different signal-to-noise ratios (SNR). When the signal-to-noise ratio is 0 , the performance of this algorithm is improved by about $40 \%$ compared to the ESPRIT-like algorithm and improved by about 25\% compared to the REC-FBSS-ESPRIT algorithm. By comparing RSMEs with different signal-to-noise ratios, it is concluded that the proposed algorithm performs better 
TABLE 1: Algorithm steps in this paper.

Step 1: construct the received data $X$ and $Y$ from different matrix models and construct the Toeplitz matrix from equations (2) and (4) Step 2: $R X i$ and $R Y i$ are obtained from Toeplitz matrix and receiving matrix through formula (7)

Step 3: select all $R X i$ and $R Y i$ to get $R X \Sigma$ and $R Y \Sigma$ from formulas (8) and (9)

Step 4: $R$ is calculated from the $R=(1 / 2)\left(R \Sigma+J R \sum^{*} J\right)$ formula

Step 5: perform feature decomposition on $R$ to obtain signal subspace $U S$

Step 6: get the corresponding $U B$ and $U F$ from $U S$, get the corresponding $\Psi$, and then perform feature decomposition Step 7: match by equation (13) to obtain two-dimensional DOA estimation of $P$ signals

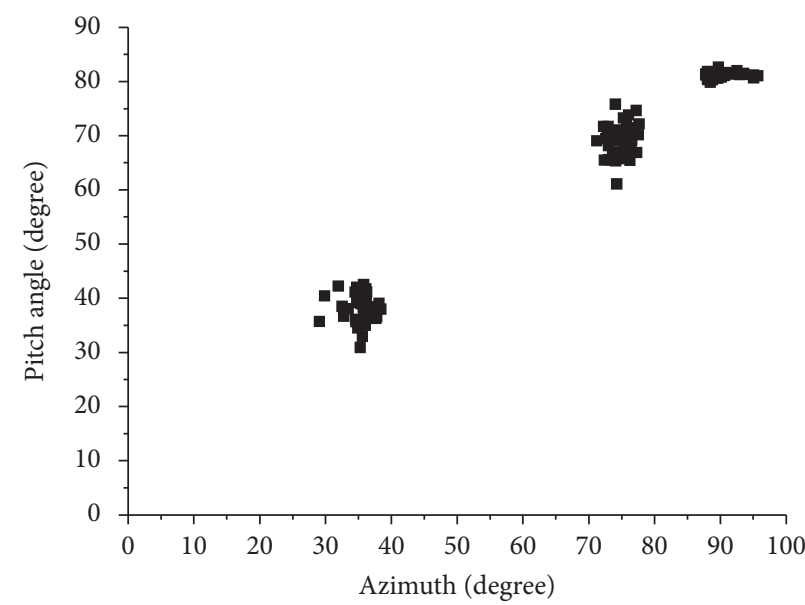

- ESPRIT-like

(a)

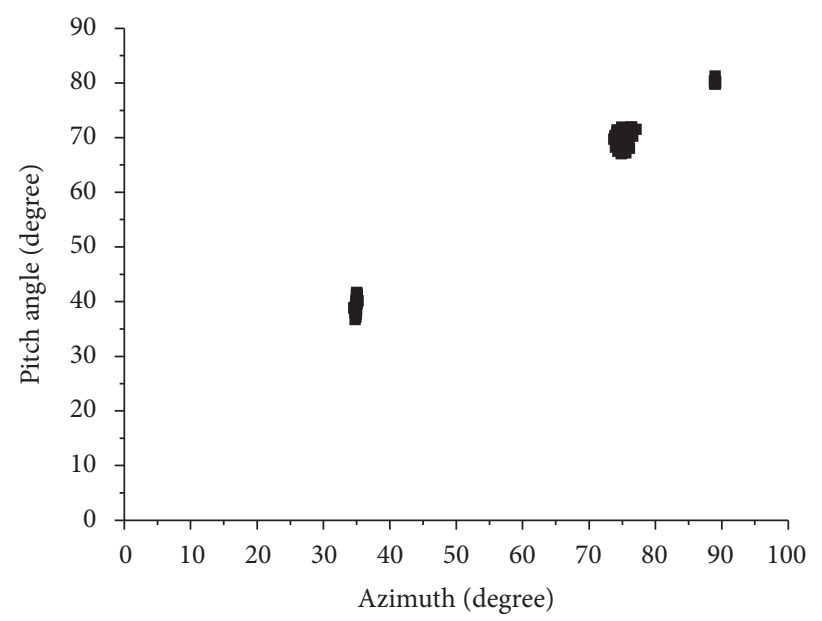

- REC-FBSS-ESPRIT

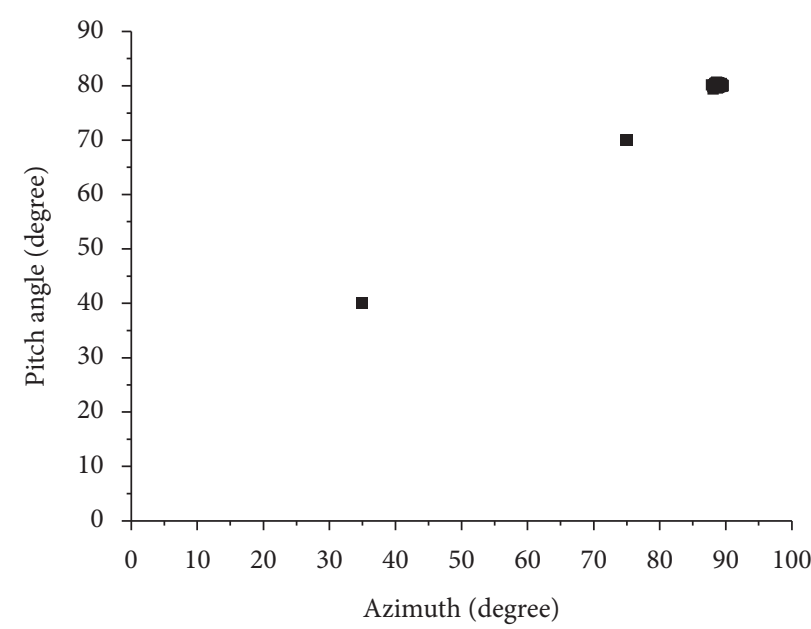

- This paper's cruciform array

(b)

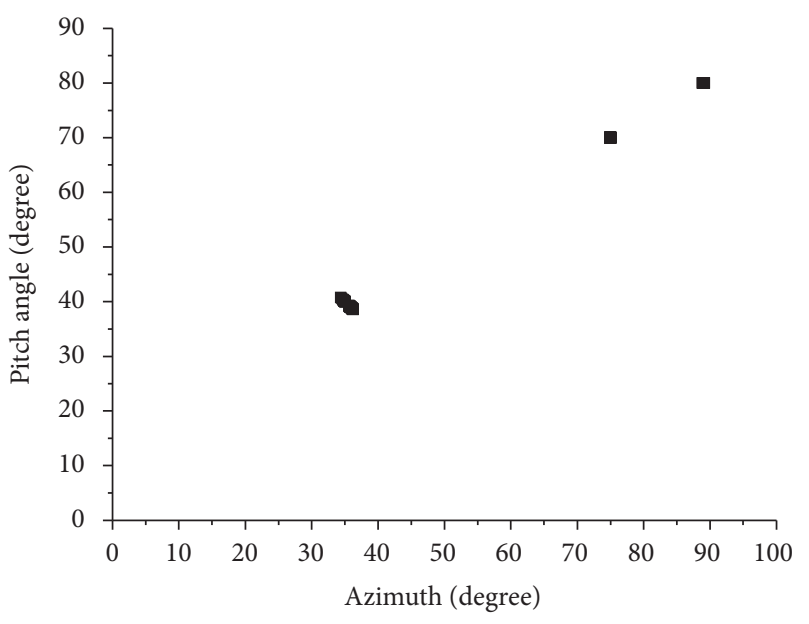

- This paper's L-type array

(d)

Figure 4: Continued. 


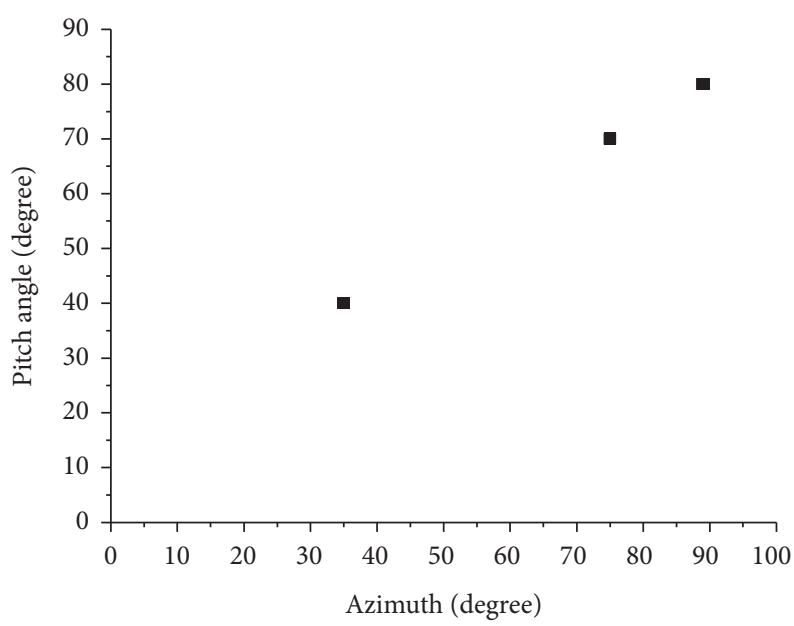

- MIMO array

(e)

FIgURE 4: DOA estimation performance of each algorithm. (a) ESPRIT-like algorithm DOA estimation performance graph. (b) Performance graph of DOA estimation of cross array in this paper. (c) REC-FBSS-ESPRIT algorithm DOA estimation performance graph. (d) The performance graph of DOA estimation of $L$-type array in this paper. (e) MIMO array DOA estimation performance graph.

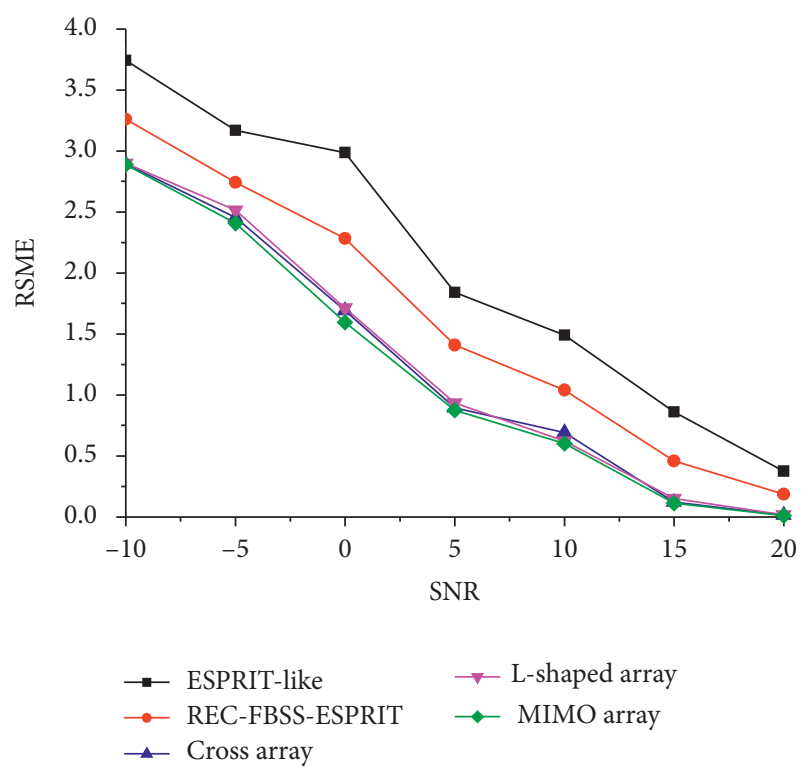

FIGURE 5: Comparison of mean square error under different signalto-noise ratios.

than the ESPRIT-like algorithm and REC-FBSS-ESPRIT algorithm at low signal-to-noise ratios.

Figure 6 shows the comparison of the resolution probability of the cross array, $L$-shaped array, MIMO array ESPRIT-like algorithm, and REC-FBSS-ESPRIT algorithm using this algorithm. Here, it is defined that if the estimated $\widehat{\theta}_{1}$ and $\widehat{\theta}_{2}$ directions of the two sources satisfy $\left|\widehat{\theta}_{1}-\theta_{1}\right|+$ $\left|\widehat{\theta}_{2}+\theta_{2}\right|<\left|\widehat{\theta}_{1}-\widehat{\theta}_{2}\right|$, it is said that the two sources are correctly resolved. The resolution probability refers to the ratio of the number of correct resolutions to the total number of experiments. The experimental conditions were 10 experiments per group under the condition that the

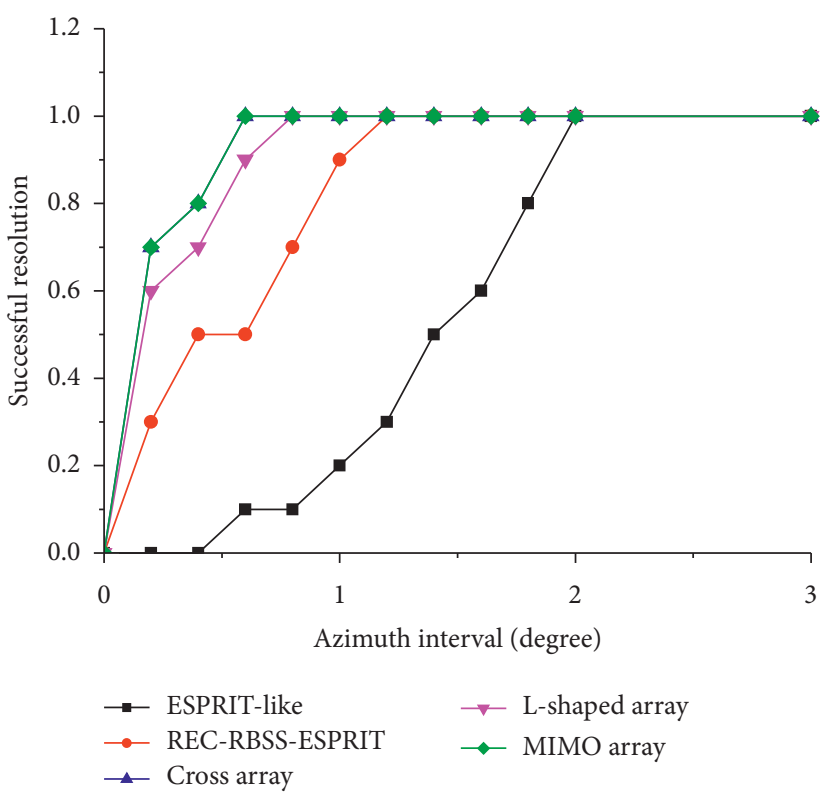

FIGURE 6: Success resolution as a function of azimuth interval.

number of snapshots was 1024 and the signal-to-noise ratio SNR was 20. The experimental results show that the probability of successful resolution of this algorithm under low azimuth interval is higher than that of ESPRIT-like algorithm and REC-FBSS-ESPRIT algorithm, which shows that the accuracy of DOA estimation of this algorithm is higher than the other two algorithms, and, moreover, the cross array, the $L$-shaped array, and the MIMO array all have better successful resolution in the case of low azimuth spacing.

Figure 7 shows the influence of correlation coefficients on the performance of each algorithm. From Figure 7(a), we 


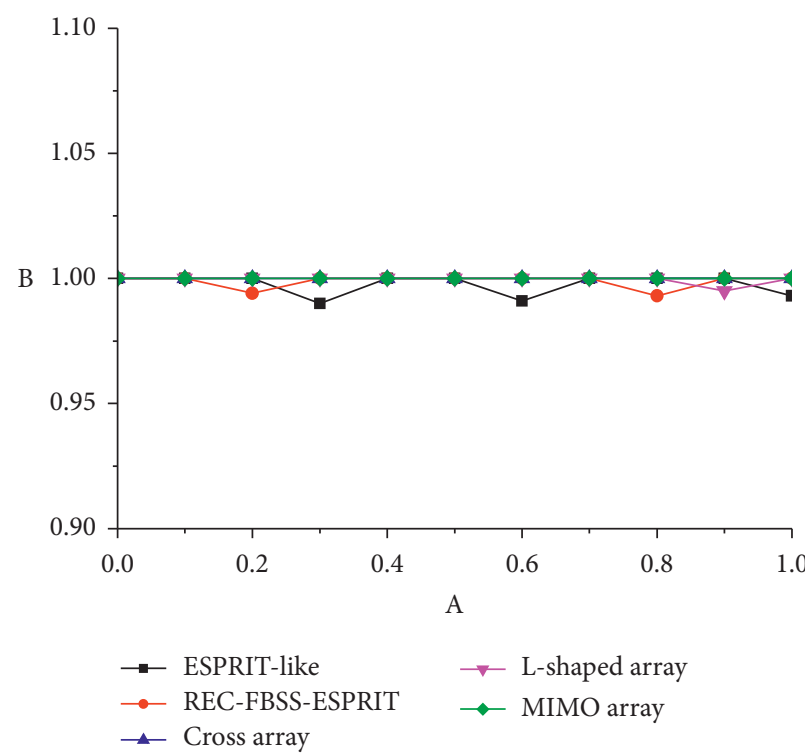

(a)

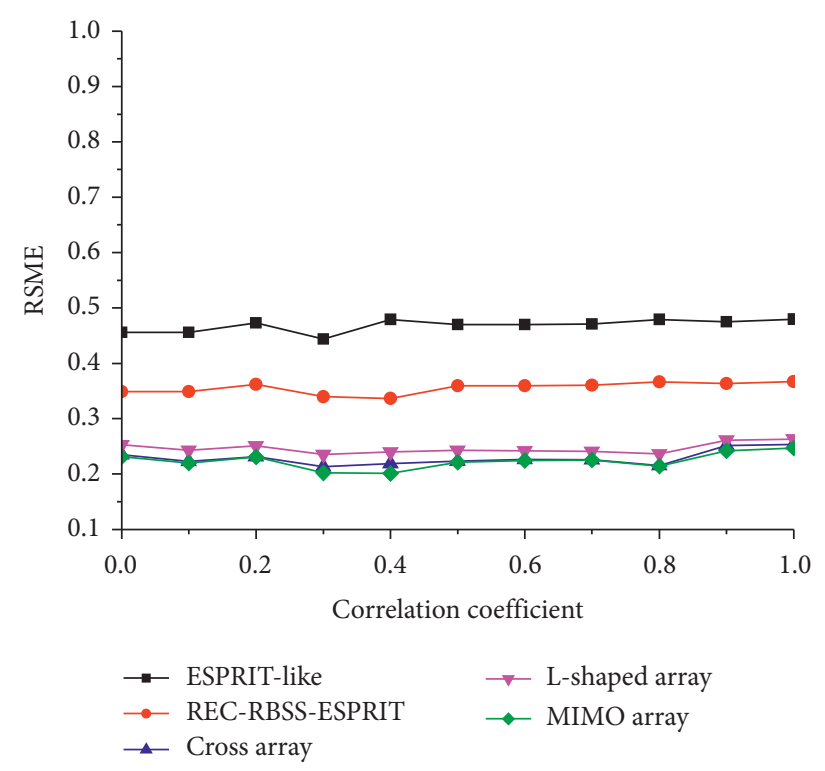

(b)

FiguRE 7: Influence of correlation coefficient on performance. (a) Relationship between the successful resolution and correlation coefficient. (b) Relationship between mean square error and correlation coefficient.

TABle 2: Main performance comparison.

\begin{tabular}{lccc}
\hline Algorithm & Snapshots: 100; SNR: 0; mean square error & $\begin{array}{c}\text { Snapshots: 20; SNR: 20; } \\
\text { mean square error }\end{array}$ & $\begin{array}{c}\text { Snapshots: 1000; SNR: 10; correlation } \\
\text { coefficient: 0.5, mean square error }\end{array}$ \\
\hline Cross array & 1.6918 & 1.0116 & 0.2335 \\
L-shaped array & 1.7132 & 1.1364 & 0.2433 \\
MIMO array & 1.832 & 1.2451 & 0.2719 \\
ESPRIT-like & 2.9868 & 1.5782 & 0.4712 \\
REC-FBSS-ESPRIT & 2.2841 & 1.2821 & 0.3594 \\
\hline
\end{tabular}

TABLE 3: DOA estimation time comparison.

\begin{tabular}{lccc}
\hline Algorithm & 100 experiments' simulation time (s) & 500 experiments' simulation time (s) & 1000 experiments simulation time (s) \\
\hline Cross array & 0.9045 & 4.1106 & 7.9423 \\
L-shaped array & 0.9769 & 4.4346 & 8.5693 \\
MIMO array & 1.0746 & 4.5571 & 8.7709 \\
ESPRIT-like & 0.7741 & 3.5709 & 7.0441 \\
REC-FBSS- & 7.9567 & 40.5768 & 96.7324 \\
ESPRIT & & &
\end{tabular}

can see the successful resolution of the MIMO array using this algorithm when the signal-to-noise ratio SNR is 10 and the number of snapshots is 1024 , and with the change of the correlation coefficient, the success resolution is always $100 \%$, and the success rate of the L-shaped array using the algorithm of this paper is only $99 \%$ to $100 \%$ when the correlation coefficient is 0.9. The success rate of the ESPRIT-like algorithm and the REC-FBSS-ESPRIT algorithm is many times between $99 \%$ and $100 \%$. It can be seen from the Figure 7 (b) that the two arrays adopting the algorithm of this paper have small changes in RSME when the correlation coefficient changes and slightly rise when the correlation coefficient is close to 1, while the ESPRIT-like algorithm has both changes when the correlation coefficient changes. The square error is also small, but the performance is lower than the algorithm in this paper. The mean square error of the REC-FBSS-ESPRIT algorithm under the change of the correlation coefficient is lower than the ESPRIT-like algorithm but higher than the algorithm in this paper. The experimental results show that the two-dimensional DOA estimation algorithm in this paper is less affected by the correlation coefficient, and the estimation performance is higher than the ESPRIT-like and REC-FBSS-ESPRIT algorithms. 
Table 2 shows the performance comparison of the cross array, L-shaped array, ESPRIT-like algorithm, and RECFBSS-ESPRIT algorithm under different parameters using this algorithm. Experiments show that the algorithm in this paper has a low signal-to-noise ratio and a low number of snapshots; the mean square error is lower than the ESPRITlike algorithm and the REC-FBSS-ESPRIT algorithm.

Table 3 shows the comparison of the time required for different algorithms to perform 100, 500, and 1000 experiments on 3 signals. The results show that the simulation time of the algorithm in this paper is similar to that of the ESPRIT-like algorithm, only slightly slower, but compared to the REC-FBSS-ESPRIT algorithm, it is much faster and has a better real-time performance.

\section{Conclusions}

This paper proposes a modified Toeplitz matrix set reconstruction algorithm based on the two-dimensional reconstruction of Toeplitz-like algorithms, which is based on the incomplete information of traditional reconstruction Toeplitz algorithm and the need for denoising. The Toeplitz matrix set is multiplied and summed with the conjugate transposed matrix to achieve solution coherence, avoiding additional denoising. The simulation experiment gives a comparative analysis of the performance of this algorithm, the ESPRIT algorithm, and REC-FBSS-ESPRIT algorithm and utilizes the special performance of MIMO arrays to simulate more arrays with fewer antennas, universality under the array model.

\section{Data Availability}

The datasets used or analysed during the current study are available from the corresponding author on reasonable request.

\section{Conflicts of Interest}

The authors declare that there are no conflicts of interest regarding the publication of this paper.

\section{Acknowledgments}

This work was supported by the National Natural Science Foundation of China under Grant no. 11574120 and Jiangsu Provincial University Natural Science Fund under Grant no. 14KJD510002.

\section{References}

[1] L. P. Qiao, C. S. I. Xi, and L. I. Li, "Fast subspace DOA algorithm without eigendecomposition," Systems Engineering and Electronics, vol. 32, no. 4, pp. 691-693, 2010.

[2] F. Mendoza-Montoya, D. H. Covarrubias-Rosales, and C. A. Lopez-Miranda, "DOA estimation in mobile communications system using subspace tracking methods," IEEE Latin America Transactions, vol. 6, no. 2, pp. 123-129, 2008.

[3] N. S. John, A. G. Konstantinos, and S. Katherine, "On the direction of arrival (DOA) estimation for a switched-beam antenna system using neural networks," IEEE Transactions on Antennas and Propagation, vol. 57, no. 5, pp. 1399-1411, 2009.

[4] J. Y. Lu and W. L. Wu, "A novel DOA algorithm for CDMA system," in Proceedings of the International Conference on Communication Technology, ICCT 2003, pp. 1164-1168, Beijing, China, 2003.

[5] J. X. Wu, T. Wang, Z. Y. Suo, and Z. Bao, "DOA estimation for ULA by spectral Capon rooting method," Electronics Letters, vol. 45, no. 1, pp. 84-85, 2009.

[6] J. Yu, W. Y. Chen, and X. F. Zhang, "Two-dimensional DOA blind estimation algorithm for coherent sources," in Proceedings of the 2009 Annual Conference on Communication Theory and Signal Processing, pp. 19-24, Quanzhou, China, 2009.

[7] C. Qian, L. Huang, M. Cao, J. Xie, and H. C. So, "PUMA: an improved realization of MODE for DOA estimation," IEEE Transactions on Aerospace and Electronic Systems, vol. 53, no. 5, pp. 2128-2139, 2017.

[8] G. Hu, H. Zhou, J. Shi et al., "Orthogonal projection method for DOA estimation in low-altitude environment based on signal subspace," AEU: Archiv fur Elektronik und Ubertragungstechnik: Electronic and Communication, vol. 83, pp. 317-321, 2018.

[9] S. F. B. Pinto and R. C. de Lamare, "Multistep knowledgeaided iterative ESPRIT: design and analysis," IEEE Transactions on Aerospace and Electronic Systems, vol. 54, no. 5, pp. 2189-2201, 2018.

[10] S. Li, Array Multi-Parameter Estimation Algorithm under the Framework of Compressed Sensing Parallel factors, Nanjing University of Aeronautics and Astronautics, Nanjing, China, 2017.

[11] R. Schmidt, "Multiple emitter location and signal parameter estimation," IEEE Transactions on Antennas \& Propagation, vol. 34, no. 5, 1986.

[12] C. Qian, L. Huang, and H. C. So, "Computationally efficient ESPRIT algorithm for direction-of-arrival estimation based on Nyström method," Signal Processing, vol. 94, pp. 74-80, 2014.

[13] C. Qian, L. Huang, W.-J. Zeng, and H. C. So, "Direction-ofArrival estimation for coherent signals without knowledge of source number," IEEE Sensors Journal, vol. 14, no. 9, pp. 3267-3273, 2014.

[14] W. Zhang, Y. Han, M. Jin, and X. L. Qiao, "Coherent source DOA estimation based on Toeplitz matrix set reconstruction," Journal of Jilin University (Engineering Science Edition), vol. 50, no. 2, pp. 703-710, 2020.

[15] J. Luo, G. Zhang, and K. Yu, "An automatically paired twodimensional direction-of-arrival estimation method for two parallel uniform linear arrays," AEU-International Journal of Electronics and Communications, vol. 72, pp. 46-51, 2017.

[16] J. Zhuang, C. Duan, W. Wang, and Z. Chen, "Joint estimation of azimuth and elevation via manifold separation for arbitrary array structures," IEEE Transactions on Vehicular Technology, vol. 67 , no. 7, pp. 5585-5596, 2018.

[17] F. M. Han and X. D. Zhang, "An ESPRIT-like algorithm for coherent DOA estimation," IEEE Antennas \& Wireless Propagation Letters, vol. 4, pp. 443-446, 2005.

[18] A. Shahimaeen and M. J. Dehghani, "Two-dimensional DOA estimation for coherent signals using a novel covariance-like matrix," Transactions on Emerging Telecommunications Technologies, vol. 30, no. 6, 2019.

[19] Y. P. Zhang, Y. L. Zhao, and X. Y. Sun, "A coherent stepwise dimension reduction DOA estimation method for $L$-shaped arrays," Journal of Computer Applications, vol. 33, no. 5, pp. 1477-1480, 2016. 